Authors' reply: As Mushtaq \& Minn-Din correctly point out, Bijl et al ${ }^{1}$ did not find associations between prevalence rates of any psychiatric disorder (including anxiety disorders) in children and anxiety-related symptoms in parents. However, one must be aware of several methodological differences to our investigation: the results cited by Mushtaq \& Minn-Din are based on 12-month prevalence rates and multivariate logistic regression analysis additionally controlling for childhood adversities and socio-demographic characteristics. We would like to clarify that the results of the Bijl et al paper are much more comparable with our study and that the results we are actually referring to are those based on life-time prevalence rates of psychiatric disorders in children without controlling for childhood adversities and reported separately for the various offspring disorders. Here, Bijl et al clearly report associations between anxiety in parents and children.

In addition, it is true that the adult children in the Bijl et al study were considerably older (18-65 years) than the offspring in our study (17-21 years at follow-up). We would like to add that there are other substantial ways in which the studies differ; for example, our use of assessment via direct interviews $v$. familyhistory information. ${ }^{1}$ Nevertheless, we do not see why our claim that we confirm and extend the Bijl et al study should be problematic, especially when taking into account the low median for age at onset of anxiety disorders. ${ }^{2}$

We would also like to point out that both studies were community-based so that the use of the term 'patients' by Mushtaq \& Minn-Din is slightly misleading.

1 Bijl RV, Cuijpers P, Smit F. Psychiatric disorders in adult children of parents with a history of psychopathology. Soc Psychiatry Psychiatr Epidemiol 2002; 37: 7-12.

2 Cross-national comparisons of the prevalences and correlates of mental disorders. WHO International Consortium in Psychiatric Epidemiology. Bull World Health Organ 2000; 78: 413-26.

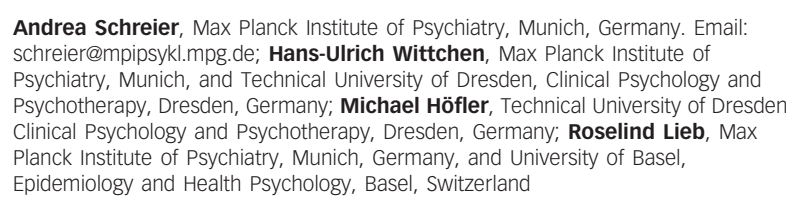

doi: 10.1192/bjp.193.1.80

\section{Cognitive-behavioural therapy for self-harm}

We read Slee et al's ${ }^{1}$ article with interest and concern. We believe there are major biases towards the treatment arm of this study which may invalidate their conclusions. Furthermore, our experience of working in a liaison psychiatry team receiving more than 1500 self-harm referrals a year leads us to question the applicability of the intervention given the characteristics of the study group.

At the outset, there are more participants in the treatmentas-usual (TAU) group shown to be depressed and this difference reaches statistical significance from the first follow-up at 3 months and gradually grows with each follow-up. Hence, it can be argued that the difference in outcome is a mere difference in depression and anxiety, which we know respond well to cognitive-behavioural therapy (CBT). Moreover, as the authors themselves admit, there was a trend from the beginning of higher suicidal cognitions in the TAU group, which assumed statistical significance from the first follow-up at 3 months. Furthermore, the authors have not attempted to match the extra time spent with participants in the CBT group with a similar amount of therapist/ contact time in the TAU group. Masking (as acknowledged) of follow-up assessments was not undertaken. Therapists in the treatment group very actively pursued participants; this may have been the active ingredient rather than CBT. Sending postcards alone as an intervention significantly reduces the frequency of hospital-treated self-poisoning events. ${ }^{2}$ All these factors bias the results in favour of the treatment group. Despite these biases, the reported benefit in reducing self-harm was marginal and only statistically significant at 9 months, with questionable clinical significance.

The participants in this study differ very significantly from the individuals seen after self-harm by routine liaison psychiatry services. The self-harm definition used was very wide, including punching and head banging, which are not usually defined as self-harm by clinicians and not proven to be associated with higher suicide risk, unlike self-poisoning and self-cutting. No data are reported on the proportion of self-harm in the study which was of this milder nature. Right from the recruitment phase, participants with alcohol and drug misuse were eliminated. This clearly skews the population enormously since a very high proportion of our patients have comorbid issues. The treatment group in particular lost eight individuals before CBT was started, and all assessments and therapy sessions were then completed. We contend that this was a highly motivated and selected group likely to benefit from the intervention, and unrepresentative of the clinical population.

Short-term interventions for self-harm have not generally proved significant when explored in large-scale studies. ${ }^{3}$ It is therefore crucial that small randomised trials of CBT or other interventions are carefully designed to minimise bias, and we feel this study fell short of the design and reporting standards we would expect. We are also concerned that high-profile publication of such studies may lead to unwarranted implementation of interventions whose effect is unproven, and whose opportunity costs are great.

1 Slee N, Garnefski N, van der Leeden R, Arensman E, Spinhoven P. Cognitivebehavioural intervention for self-harm: randomised controlled trial. $\mathrm{Br} J$ Psychiatry 2008; 192: 202-11.

2 Carter GL, Clover K, Whyte IM, Dawson AH, D'Este C. Postcards from the EDge: 24-month outcomes of a randomised controlled trial for hospitaltreated self-poisoning. Br J Psychiatry 2007; 191: 548-53.

3 Crawford MJ, Kumar P. Intervention following deliberate self-harm: enough evidence to act? Evid Based Ment Health 2007; 10: 37-9.

Mukesh Kripalani, Northern Deanery, Tees, Esk and Wear Valleys NHS Trust, Darlington, UK. Email: drmukesh@doctors.org.uk; Amanda Gash, Joe Reilly, Tees, Esk and Wear Valleys NHS Trust, Darlington, UK

doi: 10.1192/bjp.193.1.80a

Authors' reply: Kripalani et al express their concerns about biases towards the treatment arm of our study and the characteristics of our study group of patients who self-harm. With respect to biases towards the treatment arm, it should be noted that at the start of treatment no significant differences in anxiety, depression and suicidal cognitions were evident. Thus, the gradually growing difference in depression and suicidal cognitions from the first follow-up at 3 months and in anxiety at the 9-month follow-up in our opinion reflects a treatment effect. Just because the effects on secondary measures were stronger than on the target variable, we concluded that, as hypothesised, CBT primarily targeted maintaining factors of self-harm and that the specific self-harm effect was a secondary effect. Moreover, our study results remain silent on whether the treatment effects observed are attributable to specific ingredients of CBT or to the total package of CBT in addition to TAU. We agree with Kripalani et al, however, that the fact that assessments were not carried out masked to treatment 
group might have influenced outcome. With respect to characteristics of the study group, participants in our study manifested both self-poisoning (91\%) and self-injury (9\%) irrespective of the apparent purpose of the act, and therefore can be considered a representative sample of patients who self-harm. Of the contacted participants, only $7.3 \%$ were excluded because of schizophrenia or alcohol and drug misuse. Our final sample consisted of females (94\%) with a long history of self-harm (77\% reported 10 or more previous episodes of self-poisoning and/or self-injury) and severe psychological and psychiatric problems (on average four psychiatric diagnoses (mood and anxiety disorders in particular)). It is possible that CBT as an add-on to TAU is more likely to be effective for people with such chronic and severe self-harm. The fact that rate of withdrawal from CBT amounted to $17 \%$ underscores the feasibility of an intervention tailored to the needs of this particular group.

In conclusion, CBT appears to be an effective adjunct to TAU in chronic self-harm and further research on moderators and mediators of change seems warranted.

Philip Spinhoven, Unit of Clinical Psychology, Leiden University Institute for Psychological Research, and Department of Psychiatry, Leiden University Medical centre, The Netherlands. Email: spinhoven@fsw.leidenuniv.nl; Ella Arensman National Suicide Research Foundation, Cork, Ireland

doi: 10.1192/bjp.193.1.80b

\section{Virtual reality and paranoia}

The use of virtual reality to create a 'laboratory' is promising. As someone who has played computer games and has used the London underground ('tube') trains almost daily for 4 years, I was interested in the observations that those who used the tube regularly were less likely to have persecutory thinking in virtual reality, whereas an experience of playing computer games was a strong predictor of paranoid thinking. ${ }^{1}$

I am not sure whether the observations can be justified by an assumption that the game-playing individuals were reacting because they automatically processed the computer characters as real. The use of a virtual reality environment may have introduced a bias not taken into account just by estimating the duration of game play.

Cognition and automatic thoughts are based on prior experiences. Has this study taken into account how prior gaming experience may affect one's perception to a virtual reality environment, as opposed to a generalised cognition easily translated to the real world? Is there a possibility that the participants automatically processed the environment as being hostile thus making the findings 'a strong predictor of paranoid thinking' only in a virtual world?

The data provided in the paper fail to show the nature of gaming experience these people have had. Is it possible that a person who plays non-violent strategy games, or gambles online, will have a different experience of virtual reality compared with someone who plays first-person shooters where one of the primary objectives of the game would be to survive, keep safe distance and, of course, to 'kill' other players when they are in range? Also, would the findings be different if some of these people who played computer games spent their time in virtual reality social networking worlds such as 'Second Life'?

If an experience of travelling on the tube regularly shows less likelihood of feeling persecuted in a virtual train ride, can it be said that a prior experience of a threatening virtual reality environment make those who play games more likely to feel persecuted in the chosen medium than they would otherwise be in the real life?

1 Freeman D, Pugh K, Antley A, Slater M, Bebbington P, Gittins M, Dunn G, Kuipers $E$, Fowler D, Garety $P$. Virtual reality study of paranoid thinking in the general population. Br J Psychiatry 2008; 192: 258-63.
Sunanda Ghosh, Hertfordshire Partnership Foundation Trust, UK. Email: sunanda ghosh@gmail.com

doi: 10.1192/bjp.193.1.81

Freeman et al have used an innovative technique in a non-clinical population to confirm a high background prevalence of negative, mistrustful and fearful thoughts about others. ${ }^{1}$ Their paper may be helpful in encouraging healthcare professionals in their attempts to normalise rather than medicalise such thoughts, which are particularly common and pronounced in patients with neurotic and personality disorders. ${ }^{2}$

I am concerned, however, by the authors' use of the word 'paranoia' to describe these thoughts. Freeman et al define paranoia as 'the unfounded fear that others intend to cause you harm', with reference only to an earlier publication by the main author; later in the paper the words 'persecutory' and 'paranoid' are used synonymously. This definition and usage are erroneous.

Varying definitions of paranoia exist in the literature but the correct meaning of 'paranoid' is 'delusional.' ${ }^{3}$ With a Greek derivation and a literal meaning of 'out of the mind', German psychiatrists revived the term in the mid-19th century to describe conditions characterised by delusions, not only of persecution but also of grandeur. ${ }^{4}$ Later, Kraepelin, Bleuler and others variously attempted to classify paranoia, but central to all concepts was that it referred only to delusional rather than non-delusional ideation, and could include grandiose, jealous or somatic, as well as persecutory, delusions. ${ }^{4}$ Indeed, the 'paranoid' subtype of schizophrenia, still in use, refers to an illness dominated by hallucinations and delusions, and the latter need not be persecutory in nature. $^{5}$

Of course, over the 20th century, the word has taken on an entirely different meaning outside psychiatry. Anecdotally, patients frequently report 'paranoia' as an unpleasant presenting complaint, despite the fact that, by its very nature, a fixed false belief cannot be viewed by its sufferer as a symptom. Similarly, mental health professionals commonly use the term erroneously, sometimes resulting in non-psychotic patients being inappropriately referred to specialist services for those with psychosis. I fear that Freeman et al's rejection of the longstanding psychiatric definition of paranoia, in favour of its lay meaning, will only add to this unnecessary confusion.

1 Freeman D, Pugh K, Antley A, Slater M, Bebbington P, Gittins M, Dunn G, Kuipers E, Fowler D, Garety P. Virtual reality study of paranoid thinking in the general population. Br J Psychiatry 2008; 192: 258-63.

2 Reid WH, Thorne SA. Personality disorders and violence potential. J Psychiatr Pract 2007; 13: 261-8.

3 Hamilton M (ed). Fish's Clinical Psychopathology (2nd edn). ButterworthHeinemann, 1985.

4 Gelder M, Gath D, Mayou R, Cowen P. Oxford Textbook of Psychiatry (2nd edn). Oxford University Press, 1996

5 World Health Organization. The ICD-10 Classification of Mental and Behavioural Disorders: Clinical Descriptions and Diagnostic Guidelines. WHO, 1992.

Richard Braithwaite, Portsmouth City Teaching Primary Care Trust, Cavendish House, 18 Victoria Road, South Southsea, Hampshire PO5 2BZ, UK. Email: richard.braithwaite@ports.nhs.uk

doi: 10.1192/bjp.193.1.81a

Author's reply: All too often the presence of paranoid thinking has only been given significance in relation to diagnosing illness. It has been viewed as a symptom that leads to a diagnosis and that, more or less, is the end of it. An alternative view is that the experience itself should take centre stage. ${ }^{1,2}$ Persecutory thinking is 